https://helda.helsinki.fi

\title{
On universal operators and universal pairs
}

\section{Schroderus, Riikka}

2018-08

Schroderus , R \& Tylli , H-O 2018 , ' On universal operators and universal pairs ' ,

Proceedings of the Edinburgh Mathematical Society , vol. 61, no. 3 , pp. 891-908 . https://doi.org/10.1017/S001309

http://hdl.handle.net/10138/312411

https://doi.org/10.1017/S0013091518000068

acceptedVersion

Downloaded from Helda, University of Helsinki institutional repository.

This is an electronic reprint of the original article.

This reprint may differ from the original in pagination and typographic detail.

Please cite the original version. 


\title{
ON UNIVERSAL OPERATORS AND UNIVERSAL PAIRS
}

\author{
RIIKKA SCHRODERUS AND HANS-OLAV TYLLI
}

\begin{abstract}
We study some basic properties of the class of universal operators on Hilbert space, and provide new examples of universal operators and universal pairs.
\end{abstract}

\section{INTRODUCTION}

Let $\mathcal{L}(H)$ be the algebra of bounded linear operators on the separable infinite-dimensional Hilbert space $H$. Recall that the operators $T_{1} \in \mathcal{L}\left(H_{1}\right)$ and $T_{2} \in \mathcal{L}\left(H_{2}\right)$ are similar if there exists a linear isomorphism $J: H_{1} \longrightarrow H_{2}$ such that $T_{1}=J^{-1} T_{2} J$. The operator $U \in \mathcal{L}(H)$ is called universal if for any $T \in \mathcal{L}(H)$ there exist a closed $U$-invariant subspace $M \subset H$, i.e. $U(M) \subset M$, and a constant $c \neq 0$ such that the operators $U_{\mid M}: M \longrightarrow M$ and $c T: H \longrightarrow H$ are similar.

The concept of a universal operator was introduced by Rota [28], where he showed that the backward shift of infinite multiplicity is an explicit example of these seemingly strange objects. The invariant subspace problem provides motivation for studying concrete universal operators, namely, every operator in $\mathcal{L}(H)$ has a non-trivial invariant subspace if and only if the minimal non-trivial invariant subspaces of (any) universal operator are 1-dimensional. General references for this approach and results about universal operators are [3, Chapter 8] and the survey [6].

Later Caradus [1] (see also [3, Theorem 8.1.3]) exhibited a simple sufficient condition for an operator to be universal, namely,

(C) If $U \in \mathcal{L}(H)$ is such that the kernel $\operatorname{Ker}(U)$ is infinite-dimensional and its range $\operatorname{Ran}(U)=H$, then $U$ is universal for $H$.

However, Caradus' condition and its recent generalisation by Pozzi [26] are very far from being necessary. In Section 2 of this paper we look more closely at some fundamental properties of the class of universal operators, as well as some of its subclasses. In particular, we derive spectral theoretic consequences of universality which can be used to verify that a given operator is not universal.

Because of its simplicity, condition (C) is often used to obtain examples of universal operators, even though the desired properties can be difficult to verify for many concrete operators. By a celebrated example of Nordgren, Rosenthal and Wintrobe [24] from 1987 the operators $C_{\varphi}-\lambda I$ are universal on the Hardy space $H^{2}(\mathbb{D})$, whenever the composition operator $f \longmapsto C_{\varphi} f=f \circ \varphi$ is induced by a hyperbolic automorphism $\varphi$ of the unit disc $\mathbb{D}$ and $\lambda$ belongs to the interior of the spectrum of $C_{\varphi}$. In this case the infinite-dimensionality of $\operatorname{Ker}\left(C_{\varphi}-\lambda I\right)$ is verified by explicit computation, but the original argument for the surjectivity relies on fairly sophisticated properties of multiplication operators induced by certain

2010 Mathematics Subject Classification. 47B37.

Key words and phrases. Hilbert space, universal operator, universal commuting pair, composition operator. First author is supported by the Magnus Ehrnrooth Foundation in Finland. 
Blaschke products in $H^{2}(\mathbb{D})$. Only very recently an alternative argument for the universality of $C_{\varphi}-\lambda I$ on $H^{2}(\mathbb{D})$ was given in [8]. For other concrete examples of universal operators, see e.g. $[25,26,5,7]$. Moreover, the connection between the invariant subspace problem and universality has motivated recent work on the lattice of invariant subspaces of $C_{\varphi}$ on $H^{2}(\mathbb{D})$ for hyperbolic automorphisms $\varphi$, see e.g. [18] and [12].

In Section 3 we show that the adjoint $C_{\varphi}^{*}-\bar{\lambda} I$ is universal on $S^{2}(\mathbb{D})$, the Hilbert space consisting of analytic functions $f: \mathbb{D} \longrightarrow \mathbb{C}$ such that $f^{\prime} \in H^{2}(\mathbb{D})$, whenever $\lambda$ is an interior point of the spectrum of $C_{\varphi}$ on $S^{2}(\mathbb{D})$. It follows from known results that $C_{\varphi}-\lambda I$ is not a universal operator on $S^{2}(\mathbb{D})$, for any $\lambda \in \mathbb{C}$, which suggests that universality passes to the adjoint for small enough spaces in the scale of weighted Dirichlet spaces of analytic functions on $\mathbb{D}$.

Recently Müller [22] introduced a notion of universality for commuting $n$-tuples of operators, and he obtained versions of the sufficient condition $(\mathrm{C})$ in this setting. However, examples of universal commuting $n$-tuples are rather more difficult to exhibit compared to the case of a single operator, and in Section 4 we discuss new concrete examples of universal commuting pairs $\left(U_{1}, U_{2}\right) \in \mathcal{L}(H)^{2}$. In particular, we show that certain pairs $\left(L_{A}, R_{B}\right)$ of left and right multiplication operators on the ideal of the Hilbert-Schmidt operators are universal and consider the case of universal NRW-pairs $\left(C_{\varphi}-\lambda I, C_{\psi}-\mu I\right)$ in $\mathcal{L}\left(H^{2}(\mathbb{D})\right)^{2}$.

\section{Structure of the Class of Universal operators}

The main interest has been in exhibiting and analysing concrete examples of universal operators belonging to various classes of operators, and less attention has been paid to general properties of the full class

$$
\mathcal{U}(H)=\{U \in \mathcal{L}(H): U \text { is universal }\} .
$$

In this section we systematically consider $\mathcal{U}(H)$ and some of its subclasses. Clearly $\mathcal{U}\left(H_{1}\right)$ and $\mathcal{U}\left(H_{2}\right)$ are related by similarity whenever $H_{1}$ and $H_{2}$ are separable infinite-dimensional Hilbert spaces, so the particular realisation of the Hilbert space $H$ is immaterial. We will use the notation $B_{\infty}:\left(\oplus_{\mathbb{Z}_{+}} \ell^{2}\right)_{\ell^{2}} \rightarrow\left(\oplus_{\mathbb{Z}_{+}} \ell^{2}\right)_{\ell^{2}}$ for Rota's universal model operator,

$$
B_{\infty} x=B_{\infty}\left(x_{0}, x_{1}, \ldots\right)=\left(x_{1}, x_{2}, \ldots\right),
$$

for $x=\left(x_{n}\right)_{n \in \mathbb{Z}_{+}} \in\left(\oplus_{\mathbb{Z}_{+}} \ell^{2}\right)_{\ell^{2}}$, where $x_{n} \in \ell^{2}$ for any $n \geq 0$. The universality of the backward shift $B_{\infty}$ of infinite multiplicity on $\left(\oplus_{\mathbb{Z}_{+}} \ell^{2}\right)_{\ell^{2}}$ is immediate from $(\mathrm{C})$, but the original argument by Rota [28] is quite direct.

It was pointed out in $[6, \mathrm{p} .44]$ that the precise relationship between universality and condition (C) is somewhat circular: $U \in \mathcal{U}(H)$ if and only if there is a $U$-invariant infinitedimensional subspace $M \subset H$ so that the restricted operator $U_{\mid M}: M \rightarrow M$ satisfies condition (C). This is seen by recalling that the restriction of any $U \in \mathcal{U}(H)$ to some invariant subspace is similar to $c B_{\infty}$ for some $c \neq 0$, combined with an observation of Pozzi recalled separately as Proposition 2.1 below. To state the proposition in a convenient form we write operators $V \in \mathcal{L}(H)$ with respect to direct sum decompositions $H=M \oplus M^{\perp}$ as vector-valued operator matrices

$$
V=\left(\begin{array}{ll}
V_{1,1} & V_{1,2} \\
V_{2,1} & V_{2,2}
\end{array}\right)
$$

in the obvious fashion. Thus $V_{1,1}=P V_{\mid M}, V_{1,2}=P V_{\mid M^{\perp}}, V_{2,1}=Q V_{\mid M}$ and $V_{2,2}=Q V_{\mid M^{\perp}}$, where $P: H \rightarrow M$ and $Q=I-P: H \rightarrow M^{\perp}$ are the related orthogonal projections. The 
following fact allows to construct examples of universal operators having different properties on direct sums $H \oplus H$.

Proposition 2.1. ([26, Remark 1.4]) Let $H=M \oplus M^{\perp}$, where $M \subset H$ is an infinitedimensional subspace, and let

$$
V=\left(\begin{array}{cc}
U & A \\
0 & B
\end{array}\right) \in \mathcal{L}(H)
$$

as above. Suppose that $U \in \mathcal{L}(M)$ is a universal operator for $M$. Then $V \in \mathcal{U}(H)$ for any operators $A$ and $B$.

Proof. If $T \in \mathcal{L}(H)$ is given there is, by assumption, a $U$-invariant subspace $N \subset M$, and $c \neq 0$ such that $U_{\mid N}: N \rightarrow N$ and $c T: H \rightarrow H$ are similar. Fix an isometry $J_{0}: M \rightarrow H$. We have that $U_{\mid N}$ is similar to $c J_{0}^{-1} T J_{0}$. Since $N \subset M$ we get that $N$ is $V$-invariant, $V_{\mid N}=U_{\mid N}$ is similar to $c J_{0}^{-1} T J_{0}$ and consequently also to $c T$.

We are interested in conditions that enable us to decide whether a given concrete operator is universal or not, and we first consider spectral criteria. Let $\sigma(S ; H)$ denote the spectrum of $S \in \mathcal{L}(H)$. The spectrum of a diagonal sum of operators on $H_{1} \oplus H_{2}$ satisfies

$$
\sigma\left(\left(\begin{array}{cc}
U & 0 \\
0 & B
\end{array}\right) ; H_{1} \oplus H_{2}\right)=\sigma\left(U ; H_{1}\right) \cup \sigma\left(B ; H_{2}\right)
$$

for any $U \in \mathcal{L}\left(H_{1}\right)$ and $B \in \mathcal{L}\left(H_{2}\right)$. It follows from Proposition 2.1 that there is no general characterisation of universal operators purely in terms of their spectra. Nevertheless, the universality of $U \in \mathcal{U}(H)$ does have relevant consequences for various subsets of the spectrum of $U$. For this recall the classes of semi-Fredholm operators

$$
\begin{aligned}
& \Phi_{-}(H)=\{S \in \mathcal{L}(H): \operatorname{dim}(H / \operatorname{Ran}(S))<\infty\}, \\
& \Phi_{+}(H)=\{S \in \mathcal{L}(H): \operatorname{dim}(\operatorname{Ker}(S))<\infty, \operatorname{Ran}(S) \text { is closed }\},
\end{aligned}
$$

where $\Phi(H)=\Phi_{+}(H) \cap \Phi_{-}(H)$ consists of the Fredholm operators. Operators $S \in \Phi_{+}(H)$ cannot be universal, since clearly $\operatorname{Ker}(S)$ has to be infinite-dimensional for $S$ to be an universal operator. We will need the $\Phi_{+}$-spectrum of $S \in \mathcal{L}(H)$ defined as

$$
\sigma_{e}^{+}(S ; H)=\left\{\lambda \in \mathbb{C}: S-\lambda I \notin \Phi_{+}(H)\right\} .
$$

It is known [21, Chapter III.19] that $\sigma_{e}^{+}(S ; H)$ is a non-empty compact subset of the essential spectrum

$$
\sigma_{e}(S ; H)=\{\lambda \in \mathbb{C}: S-\lambda I \notin \Phi(H)\}
$$

of $S$. Furthermore, let $\sigma_{p}(S ; H)$ denote the point spectrum of $S$.

It follows from the definition of universality that Riesz operators $S \in \mathcal{L}(H)$ can not be universal. (Recall that $S$ is a Riesz operator if $\sigma_{e}(S ; H)=\{0\}$.) The following result reveals some further common spectral properties of universal operators.

Theorem 2.2. Let $U \in \mathcal{U}(H)$ be an arbitrary universal operator. Then the following hold:

(i) There is $r>0$ such that the open disk

$$
B(0, r) \subset \sigma_{p}(U ; H) \cap \sigma_{e}^{+}(U ; H) \subset \sigma_{e}(U ; H) \subset \sigma(U ; H),
$$

and, moreover, any $\lambda \in B(0, r)$ is an eigenvalue of $U$ having infinite multiplicity. In particular, if $U \in \mathcal{U}(H)$ then 0 is an interior point of any of the sets $\sigma_{e}^{+}(U ; H)$, $\sigma_{e}(U ; H), \sigma_{p}(U ; H)$ as well as $\sigma(U ; H)$. 
(ii) There is $r>0$ and a vector-valued holomorphic map $z \mapsto y_{z}: B(0, r) \rightarrow H$ for which

$$
U y_{z}=z y_{z}, \quad z \in B(0, r) .
$$

Proof. We first recall some well-known spectral properties of the backward shift $B_{\infty}$ on the direct sum $H_{0} \equiv\left(\oplus_{N} \ell^{2}\right)_{\ell^{2}}$. Let $|z|<1$ and fix the non-zero vector $x_{0} \in \ell^{2}$, whence the sequence $x_{z}=\left(z^{n} x_{0}\right)_{n \geq 0}=\left(x_{0}, z x_{0}, \ldots\right) \in H_{0}$. Clearly

$$
B_{\infty}\left(x_{z}\right)=\left(z x_{0}, z^{2} x_{0}, \ldots\right)=z x_{z},
$$

so that any $|z|<1$ is an eigenvalue of infinite multiplicity for $B_{\infty}$, that is $z \in \sigma_{e}^{+}\left(B_{\infty} ; H_{0}\right)$. Moreover, $z \mapsto x_{z}$ is a (weakly) holomorphic map $B(0,1) \rightarrow H_{0}$, since

$$
z \mapsto\left\langle x_{z}, y\right\rangle=\sum_{n=0}^{\infty} z^{n}\left\langle x_{0}, y_{n}\right\rangle
$$

is analytic for any $y=\left(y_{n}\right) \in H_{0}$, where $\langle\cdot, \cdot\rangle$ denotes the respective inner-product.

Let $U$ be an arbitrary universal operator on $H$. By assumption there is a constant $c \neq 0$, a $U$-invariant subspace $M \subset H$ and a linear isomorphism $J: H_{0} \rightarrow M$ so that $U_{\mid M}=$ $J\left(c B_{\infty}\right) J^{-1}$. Since eigenvalues are preserved under similarity we get that

$$
B(0,|c|) \subset \sigma_{p}\left(c B_{\infty} ; H_{0}\right)=\sigma_{p}\left(U_{\mid M} ; M\right) \subset \sigma(U ; H) .
$$

Towards the related claim for $\sigma_{e}^{+}(U ; H)$ one obtains instead that

$$
B(0,|c|) \subset \sigma_{e}^{+}\left(c B_{\infty} ; H_{0}\right)=\sigma_{e}^{+}\left(U_{\mid M} ; M\right) \subset \sigma_{e}^{+}(U ; H) .
$$

For the right-hand inclusion note e.g. that $\operatorname{Ker}\left(\lambda I_{M}-U_{\mid M}\right) \subset \operatorname{Ker}(\lambda I-U)$, where the left-hand subspace is infinite-dimensional by similarity, since $\operatorname{dim}\left(\operatorname{Ker}\left(\lambda I-c B_{\infty}\right)\right)=\infty$.

Finally, the above identities $B_{\infty} x_{z}=z x_{z}$ and $c J B_{\infty}=\left(U_{\mid M}\right) J$ imply that

$$
U\left(J x_{z}\right)=c J B_{\infty}\left(x_{z}\right)=c z J\left(x_{z}\right), \quad|z|<1 .
$$

It follows that the renormalised holomorphic map $z \mapsto y_{z} \equiv J\left(x_{z / c}\right)$ satisfies condition (ii) in the disk $B(0,|c|)$.

By Example 3.2 below the spectral condition (1) in Theorem 2.2 is not sufficient for the universality of $U$. We next state some typical applications of the preceding result.

Corollary 2.3. The operator $T \in \mathcal{L}(H)$ can not be universal if any of the following conditions holds:

(i) the interior $\operatorname{int}\left(\sigma_{p}(T ; H)\right)=\emptyset$,

(ii) the interior $\operatorname{int}\left(\sigma_{e}(T ; H)\right)=\emptyset$,

(iii) every non-zero eigenvalue $\alpha \in \sigma_{p}(T ; H)$ has finite multiplicity.

Another immediate consequence of Theorem 2.2 which will be useful in Section 3 reads as follows.

Corollary 2.4. Suppose that $T \in \mathcal{L}(H)$ and $\lambda \in \partial \sigma(T ; H)$. Then $T-\lambda I$ can not be universal. In particular, if $\sigma(T ; H)=\partial \sigma(T ; H)$, then $T-\lambda I$ is not universal for any $\lambda \in \mathbb{C}$.

We next consider general properties of the class $\mathcal{U}(H)$ of the universal operators. Recently Pozzi [26, Thm. 3.8] extended Caradus' condition (C) as follows:

$\left(\mathrm{C}_{+}\right)$If $U \in \mathcal{L}(H)$ satisfies $\operatorname{dim} \operatorname{Ker}(U)=\infty$ and $\operatorname{dim}(H / \operatorname{Ran}(U))<\infty$, then $U \in \mathcal{U}(H)$. 
It is helpful for comparative purposes to introduce the subclasses

$$
\begin{aligned}
\mathcal{U} C(H) & =\{U \in \mathcal{L}(H): U \text { satisfies }(C)\}, \\
\mathcal{U} C_{+}(H) & =\left\{U \in \mathcal{L}(H): U \text { satisfies }\left(C_{+}\right)\right\}
\end{aligned}
$$

of $\mathcal{U}(H)$. Observe that $\mathcal{U} C_{+}(H)=\Phi_{-}(H) \backslash \Phi_{+}(H)$. Hence it follows from the classical perturbation theory for semi-Fredholm operators that $\mathcal{U} C_{+}(H)$ is preserved under sufficiently small operator norm perturbations as well as compact perturbations, see [2, Thm. 4.2] or [21, Thms. III.16.18 and III.16.19]. In particular, $U+K \in \mathcal{U} C_{+}(H)$ whenever $U \in \mathcal{U} C(H)$ and $K$ is a compact operator. In the sequel, we denote by $\mathcal{K}(H)$ the closed ideal of $\mathcal{L}(H)$ consisting of compact operators on $H$. Moreover, in [5, Thm. 2] the authors obtained by direct means a perturbation result for the class $\mathcal{U} C(H)$ which contains more detailed information. We also recall that the universal model operator $B_{\infty}$ has the stronger property that its restrictions represent suitable multiples $c T$ up to unitary equivalence for any $T \in \mathcal{L}(H)$, see e.g. [9, Thm. 8.1.5] or [27, Chapt. 1.5].

It is evident from Proposition 2.1 that the subclasses $\mathcal{U} C(H)$ and $\mathcal{U} C_{+}(H)$ are much smaller than $\mathcal{U}(H)$, and $\mathcal{U}(H)$ contains operators very different from $B_{\infty}$. Moreover, $\mathcal{U} C(H)$ is not preserved by compact perturbations. For the record we include related very simple examples.

Example 2.1. (i) Let $U \in \mathcal{U}(H)$, so that

$$
V=\left(\begin{array}{cc}
U & 0 \\
0 & B
\end{array}\right)
$$

is a universal operator on $H \oplus H$ for any $B \in \mathcal{L}(H)$ by Proposition 2.1. For instance, if $B(H)$ has infinite codimension in $H$, then $\operatorname{Ran}(V)=U(H)+B(H)$ has infinite codimension in $H \oplus H$, and if $\operatorname{Ran}(B)$ is not closed, then $\operatorname{Ran}(V)$ is not closed either.

(ii) Define $U \in \mathcal{L}\left(\ell^{2}\right)$ by $U e_{2 n}=e_{n}$ and $U e_{2 n+1}=0$ for $n \in \mathbb{N}$, so that $U \in \mathcal{U} C\left(\ell^{2}\right)$. Let $K \in \mathcal{K}\left(\ell^{2}\right)$ be the rank-1 operator defined by $K e_{2}=-e_{1}$ and $K e_{n}=0$ for $n \neq 2$. Since $(U+K) e_{2}=0$ it follows that $e_{1} \notin(U+K)\left(\ell^{2}\right)$, so that $U+K \notin \mathcal{U} C\left(\ell^{2}\right)$ (even though $\left.U+K \in \mathcal{U} C_{+}\left(\ell^{2}\right)\right)$.

More significantly, explicit examples demonstrate similarly that the full class $\mathcal{U}(H)$ of universal operators is neither open in the operator norm nor invariant under compact perturbations.

Example 2.2. Fix a universal operator $U \in \mathcal{U}(H)$. Proposition 2.1 implies that

$$
V=\left(\begin{array}{cc}
U & I_{H} \\
0 & 0
\end{array}\right)
$$

is a universal operator on $H \oplus H$, where $I_{H}$ is the identity map of $H$. Consider the sequence $\left(V_{n}\right) \subset \mathcal{L}(H \oplus H)$ defined by

$$
V_{n}=\left(\begin{array}{cc}
U & I_{H} \\
\frac{1}{n} I_{H} & 0
\end{array}\right)
$$

that is, $V_{n}(x, y)=\left(U x+y, \frac{1}{n} x\right)$ for $(x, y) \in H \oplus H$. Note that $V_{n}$ is not universal on $H \oplus H$ for any $n \in \mathbb{N}$, since $\operatorname{Ker}\left(V_{n}\right)=\{(0,0)\}$. In fact, $V_{n}(x, y)=\left(U x+y, \frac{1}{n} x\right)=(0,0)$ yields that $x=0$ and $y=-U x=0$. Clearly $\left\|V_{n}-V\right\|=\frac{1}{n}$ for $n \in \mathbb{N}$, so that $V \in \mathcal{U}(H \oplus H)$ is not an interior point of $\mathcal{U}(H \oplus H)$. 
Furthermore, let $K \in \mathcal{K}(H)$ be the diagonal operator defined by $K f_{n}=\frac{1}{n} f_{n}$ for $n \in \mathbb{N}$, where $\left(f_{n}\right)$ is some fixed orthonormal basis of $H$. Consider

$$
W=\left(\begin{array}{cc}
U & I_{H} \\
K & 0
\end{array}\right)=V+\left(\begin{array}{cc}
0 & 0 \\
K & 0
\end{array}\right) \in \mathcal{L}(H \oplus H),
$$

that is, $W(x, y)=(U x+y, K x)$ for $(x, y) \in H \oplus H$. Thus $W$ is a compact perturbation of $V \in \mathcal{U}(H \oplus H)$, but $W$ is not a universal operator, since again $\operatorname{Ker}(W)=\{(0,0)\}$.

It follows from the algebraic semi-group property of $\Phi_{-}(H)$, see [21, Thm. III.16.5], that the subclass $\mathcal{U} C_{+}(H)$ is multiplicative in the sense that $U V \in \mathcal{U} C_{+}(H)$ whenever $U, V \in \mathcal{U} C_{+}(H)$ (and this property is obvious for $\mathcal{U} C(H)$ ). Multiplicativity easily fails for the class $\mathcal{U}(H)$. In fact, fix $U_{0}, V_{0} \in \mathcal{U}(H)$. Then $U=\left(\begin{array}{cc}U_{0} & 0 \\ 0 & 0\end{array}\right)$ and $V=\left(\begin{array}{cc}0 & 0 \\ 0 & V_{0}\end{array}\right)$ belong to $\mathcal{U}(H \oplus H)$ by Proposition 2.1, but $U V=0$.

\section{Universality of the ADJoint $C_{\varphi}^{*}-\bar{\lambda} I$ ON $S^{2}(\mathbb{D})$}

Recall that Nordgren, Rosenthal and Wintrobe [24] showed that the operators $C_{\varphi}-\lambda I$ are universal on the Hardy space $H^{2}(\mathbb{D})$ for any hyperbolic automorphism $\varphi$ of the unit disc $\mathbb{D}$ and $\lambda \in \operatorname{int}\left(\sigma\left(C_{\varphi} ; H^{2}(\mathbb{D})\right)\right)$. Here $C_{\varphi}$ is the composition operator $f \mapsto f \circ \varphi$. In this section we will discuss potential analogues of this result in the scale of weighted Dirichlet spaces $D_{\beta}(\mathbb{D})$, which are Hilbert spaces of analytic functions defined on the unit disc $\mathbb{D}$. Our main observation (Theorem 3.1) is that the adjoint $C_{\varphi}^{*}-\bar{\lambda} I$ is universal on the space $S^{2}(\mathbb{D})$, whenever $\varphi$ is a hyperbolic automorphism of $\mathbb{D}$ and $\lambda \in \operatorname{int}\left(\sigma\left(C_{\varphi} ; S^{2}(\mathbb{D})\right)\right)$. Here $S^{2}(\mathbb{D})$ is the Hilbert space consisting of the analytic functions $f: \mathbb{D} \longrightarrow \mathbb{C}$ such that $f^{\prime} \in H^{2}(\mathbb{D})$, whence $S^{2}(\mathbb{D})$ is continuously embedded in the classical Dirichlet space $\mathcal{D}^{2}$.

Recall for $\beta \in \mathbb{R}$ that the weighted Dirichlet space $\mathcal{D}_{\beta}(\mathbb{D})$ is the Hilbert space of analytic functions $f(z)=\sum_{n=0}^{\infty} a_{n} z^{n}$ that satisfy

$$
\|f\|_{\beta}^{2}=\sum_{n=0}^{\infty}\left|a_{n}\right|^{2}(n+1)^{2 \beta}<\infty .
$$

(These spaces are also special cases of the weighted Hardy spaces.) The Hardy space $H^{2}(\mathbb{D})$ is obtained for $\beta=0$, the Bergman space $A^{2}(\mathbb{D})$ for $\beta=-1 / 2$, the Dirichlet space $\mathcal{D}^{2}$ for $\beta=1 / 2$ and $S^{2}(\mathbb{D})$ for $\beta=1$ (possibly up to an equivalent norm). We also recall that there is a continuous embedding $D_{\beta}(\mathbb{D}) \subset D_{\alpha}(\mathbb{D})$ for $\alpha<\beta$. The reference [9, Chapter 2.1] contains more background about these spaces.

It will be enough for our purposes to consider the normalized hyperbolic automorphisms of $\mathbb{D}$ that have the form

$$
\varphi_{r}(z)=\frac{z+r}{1+r z}, \quad r \in(0,1) .
$$

In fact, it is known that all other hyperbolic automorphisms of $\mathbb{D}$ can be conjugated by automorphisms of $\mathbb{D}$ to the preceding normalised form. We will later need the fact that

$$
\varphi_{r}^{-1}(z)=\varphi_{-r}(z)=\frac{z-r}{1-r z}
$$

belongs to the same conjugacy class as $\varphi_{r}$, since $\varphi_{-r}=g \circ \varphi_{r} \circ g$, where $g(z)=-z$ for $z \in \mathbb{D}$. Hence $C_{\varphi_{-r}}=C_{g} C_{\varphi_{r}} C_{g}$, so that $C_{\varphi_{r}}$ and $C_{\varphi_{-r}}=C_{\varphi_{r}}^{-1}$ are similar operators. For more 
information on linear fractional transformations in general, see e.g. [31, Chapter 0], and on composition operators acting on spaces of analytic functions, see [9] or [31].

The composition operators $C_{\varphi_{r}}$ are known to be bounded on $\mathcal{D}_{\beta}(\mathbb{D})$ for all $\beta \in \mathbb{R}$, see $[9$, Chapter 3.1] and [32] for various ranges of $\beta$. We will require the result that

$$
\sigma\left(C_{\varphi_{r}} ; S^{2}(\mathbb{D})\right)=\sigma\left(C_{\varphi_{r}} ; H^{2}(\mathbb{D})\right)=\left\{\lambda \in \mathbb{C}:\left(\frac{1-r}{1+r}\right)^{1 / 2} \leq|\lambda| \leq\left(\frac{1-r}{1+r}\right)^{-1 / 2}\right\}
$$

for all $0<r<1$. We refer to [9, Thm. 7.4] for the Hardy space case and to [13, Thm. 3.9] for the case $S^{2}(\mathbb{D})=\mathcal{D}_{1}(\mathbb{D})$. In the sequel we will denote the corresponding open annulus, i.e. the interior of the above spectrum, by

$$
\mathcal{A}_{r}:=\left\{\lambda \in \mathbb{C}:\left(\frac{1-r}{1+r}\right)^{1 / 2}<|\lambda|<\left(\frac{1-r}{1+r}\right)^{-1 / 2}\right\} .
$$

We point out as an initial motivation that $C_{\varphi_{r}}-\lambda I$ is not universal on any of the small weighted Dirichlet spaces contained in the classical Dirichlet space $\mathcal{D}^{2}=\mathcal{D}_{1 / 2}(\mathbb{D})$.

Example 3.1. Let $0<r<1$. Then $C_{\varphi_{r}}-\lambda I$ is not universal on $\mathcal{D}_{\beta}(\mathbb{D})$ for any $\beta \geq 1 / 2$ and any $\lambda \in \mathbb{C}$.

In fact, for $\beta=1 / 2$ it is known that $\sigma\left(C_{\varphi_{r}} ; \mathcal{D}^{2}\right)=\mathbb{T}$ by [15, Thm. 3.2]. Hence it follows from Corollary 2.4 that neither $C_{\varphi_{r}}-\lambda I$ nor its adjoint $C_{\varphi_{r}}^{*}-\bar{\lambda} I$ is universal on $\mathcal{D}^{2}$ for any $\lambda \in \mathbb{C}$.

For $\beta>1 / 2$ it follows from [13, Thm. 3.9] and its proof that in this case the point spectrum $\sigma_{p}\left(C_{\varphi_{r}} ; \mathcal{D}_{\beta}(\mathbb{D})\right)=\{1\}$, and moreover that $\operatorname{Ker}\left(C_{\varphi_{r}}-I ; \mathcal{D}_{\beta}(\mathbb{D})\right)=\mathbb{C}$. Consequently $\operatorname{dim} \operatorname{Ker}\left(C_{\varphi_{r}}-\lambda I ; \mathcal{D}_{\beta}(\mathbb{D})\right)$ is either $0($ for $\lambda \neq 1$ ) or 1 (for $\lambda=1$ ), so Corollary 2.3 yields that $C_{\varphi_{r}}-\lambda I$ can not be universal on the weighted Dirichlet spaces for any $\beta>1 / 2$ and $\lambda \in \mathbb{C}$.

As a contrast we show in the main result of this section that the adjoint of $C_{\varphi_{r}}-\lambda I$ is universal on $S^{2}(\mathbb{D})$.

Theorem 3.1. Let $\varphi_{r}$ be the hyperbolic automorphism of $\mathbb{D}$ defined by $\varphi_{r}(z)=\frac{z+r}{1+r z}$ for $r \in(0,1)$. Then $C_{\varphi_{r}}^{*}-\bar{\lambda} I$ is universal on $S^{2}(\mathbb{D})$ for any $\lambda \in \mathcal{A}_{r}$.

Proof. Let $0<r<1$ and write $H^{2}(\mathbb{D})=z H^{2}(\mathbb{D}) \oplus[\mathbf{1}]$, where [1] denotes the constant functions. The crux of the argument is the fact that the compression

$$
P_{z H^{2}} C_{\varphi_{r}}^{-1}: z H^{2}(\mathbb{D}) \longrightarrow z H^{2}(\mathbb{D})
$$

and the restriction of the adjoint

$$
C_{\varphi_{r}}^{*}: z S^{2}(\mathbb{D}) \longrightarrow z S^{2}(\mathbb{D})
$$

are similar operators, where the subspace $z S^{2}(\mathbb{D})$ is invariant under $C_{\varphi_{r}}^{*}$. The details of the similarity are explained in Corollary 3.6 and Remark 3.8 in [13], which in turn is based on a duality argument of Hurst [16, Thm. 5].

We first consider the operator $C_{\varphi_{r}}^{-1}-\lambda I: H^{2}(\mathbb{D}) \longrightarrow H^{2}(\mathbb{D})$. Since $C_{\varphi_{r}}^{-1}(\mathbf{1})=\mathbf{1}$ we may write $C_{\varphi_{r}}^{-1}-\lambda I$ as the following operator matrix acting on $H^{2}(\mathbb{D})=z H^{2}(\mathbb{D}) \oplus[\mathbf{1}]$ :

$$
C_{\varphi_{r}}^{-1}-\lambda I=\left(\begin{array}{cc}
P_{z H^{2}} C_{\varphi_{r}}^{-1}-\lambda I & 0 \\
P_{\mathbb{C}} C_{\varphi_{r}}^{-1} & (1-\lambda)
\end{array}\right)
$$

We claim that the compression $P_{z H^{2}} C_{\varphi_{r}}^{-1}-\lambda I: z H^{2}(\mathbb{D}) \longrightarrow z H^{2}(\mathbb{D})$ satisfies Caradus' condition (C) for all $\lambda \in \mathcal{A}_{r}$. Since $C_{\varphi_{r}}^{-1}=C_{\varphi_{-r}}$ we know that the operator $C_{\varphi_{r}}^{-1}-\lambda I$ : 
$H^{2}(\mathbb{D}) \longrightarrow H^{2}(\mathbb{D})$ is surjective by the proof of $[24$, Thm. 6.2]. Hence it follows that $\left(P_{z H^{2}} C_{\varphi_{r}}^{-1}-\lambda I\right)\left(z H^{2}(\mathbb{D})\right)=z H^{2}(\mathbb{D})$ as well. In fact, if $g \in z H^{2}(\mathbb{D})$ is arbitrary, then there is $f=f_{1}+f_{2} \in H^{2}(\mathbb{D})$, with $f_{1} \in z H^{2}(\mathbb{D})$ and $f_{2} \in[\mathbf{1}]$, such that $\left(C_{\varphi_{r}}^{-1}-\lambda I\right) f=g$, that is

$$
\left(\begin{array}{cc}
P_{z H^{2}} C_{\varphi_{r}}^{-1}-\lambda I & 0 \\
P_{\mathbb{C}} C_{\varphi_{r}}^{-1} & (1-\lambda)
\end{array}\right)\left(\begin{array}{c}
f_{1} \\
f_{2}
\end{array}\right)=\left(\begin{array}{c}
\left(P_{z H^{2}} C_{\varphi_{r}}^{-1}-\lambda I\right) f_{1} \\
P_{\mathbb{C}} C_{\varphi_{r}}^{-1} f_{1}+(1-\lambda) f_{2}
\end{array}\right)=\left(\begin{array}{l}
g \\
0
\end{array}\right) .
$$

In particular, $\left(P_{z H^{2}} C_{\varphi_{r}}^{-1}-\lambda I\right) f_{1}=g$, so that the compression $P_{z H^{2}} C_{\varphi_{r}}^{-1}-\lambda I$ is an onto map $z H^{2}(\mathbb{D}) \longrightarrow z H^{2}(\mathbb{D})$ for $\lambda \in \mathcal{A}_{r}$. Moreover, it is not difficult to check that since $\lambda \in \mathcal{A}_{r}$ is an eigenvalue of infinite multiplicity for $C_{\varphi_{r}}^{-1}: H^{2}(\mathbb{D}) \longrightarrow H^{2}(\mathbb{D})$, the same fact holds for the compression $P_{z H^{2}} C_{\varphi_{r}}^{-1}: z H^{2}(\mathbb{D}) \longrightarrow z H^{2}(\mathbb{D})$. Consequently $P_{z H^{2}} C_{\varphi_{r}}^{-1}-\lambda I: z H^{2}(\mathbb{D}) \longrightarrow$ $z H^{2}(\mathbb{D})$ satisfies $(\mathrm{C})$.

It follows from the similarity stated in the beginning of the argument that the restricted adjoint $C_{\varphi_{r}}^{*}-\lambda I: z S^{2}(\mathbb{D}) \longrightarrow z S^{2}(\mathbb{D})$ also satisfies $(\mathrm{C})$ and is hence universal on $z S^{2}(\mathbb{D})$. Write $C_{\varphi_{r}}^{*}-\lambda I$ on $S^{2}(\mathbb{D})$ as an operator matrix acting on $z S^{2}(\mathbb{D}) \oplus[\mathbf{1}]$, that is,

$$
\left(\begin{array}{cc}
C_{\varphi_{r}}^{*}-\lambda I & P_{z S^{2}} C_{\varphi_{r}}^{*} \\
0 & (1-\lambda)
\end{array}\right)
$$

where we also take into account that $C_{\varphi_{r}}^{*}\left(z S^{2}(\mathbb{D})\right) \subset z S^{2}(\mathbb{D})$. It follows that $C_{\varphi_{r}}^{*}-\lambda I$ : $S^{2}(\mathbb{D}) \longrightarrow S^{2}(\mathbb{D})$ is universal by Proposition 2.1.

Alternatively, in the last step one may also note that if $\lambda \neq 1$, then $C_{\varphi_{r}}^{*}-\lambda I: S^{2}(\mathbb{D}) \longrightarrow$ $S^{2}(\mathbb{D})$ satisfies $(\mathrm{C})$, while codim $\operatorname{Ran}\left(C_{\varphi_{r}}^{*}-I\right)=1$ in $S^{2}(\mathbb{D})$, so that $C_{\varphi_{r}}^{*}-I$ satisfies the generalised condition $\left(\mathrm{C}_{+}\right)$.

Finally, note that the annulus $\mathcal{A}_{r}$ is preserved by complex conjugation, so that we may above change $C_{\varphi_{r}}^{*}-\lambda I$ to $C_{\varphi_{r}}^{*}-\bar{\lambda} I$.

Heller [14] found a concrete formula for the adjoint $C_{\varphi_{r}}^{*}$ on $S^{2}(\mathbb{D})$ which involves a compact perturbation. This fact leads to a related universal operator. Let $M_{z}$ be the multiplication operator $f \mapsto z f$ on $S^{2}(\mathbb{D})$, whose adjoint $M_{z}^{*} \in \mathcal{L}\left(S^{2}(\mathbb{D})\right)$ has the form

$$
M_{z}^{*}\left(\sum_{n=0}^{\infty} a_{n} z^{n}\right)=a_{1}+\sum_{n=1}^{\infty} a_{n+1}\left(\frac{n+1}{n}\right)^{n} z^{n}
$$

for $f(z)=\sum_{n=0}^{\infty} a_{n} z^{n} \in S^{2}(\mathbb{D})$.

Corollary 3.2. Let $\varphi_{r}$ be as in Theorem 3.1. Then the operator

$$
C_{\varphi_{r}}-\frac{r}{1+r^{2}}\left(M_{z}^{*}+M_{z}\right) C_{\varphi_{r}}-\frac{1-r^{2}}{1+r^{2}} \lambda I
$$

is universal on $S^{2}(\mathbb{D})$ for all $\lambda \in \mathcal{A}_{r}$.

Proof. By [14, Thm. 6.5], we can write

$$
\left(C_{\varphi_{r}}^{-1}\right)^{*}-\lambda I=\frac{1+r^{2}}{1-r^{2}} C_{\varphi_{r}}-\frac{r}{1-r^{2}}\left(M_{z}^{*}+M_{z}\right) C_{\varphi_{r}}-\lambda I+K
$$

where $K$ is a compact operator on $S^{2}(\mathbb{D})$. Recall that $C_{\varphi_{r}}$ and $C_{\varphi_{r}}^{-1}=C_{\varphi_{-r}}$ are similar operators on $S^{2}(\mathbb{D})$. From the symmetry of $\mathcal{A}_{r}$ we get that Theorem 3.1 holds if we replace $C_{\varphi_{r}}^{*}$ by $\left(C_{\varphi_{r}}^{-1}\right)^{*}$. Moreover, the proof of Theorem 3.1 shows that $\left(C_{\varphi_{r}}^{-1}\right)^{*}-\lambda I$ satisfies condition $\left(\mathrm{C}_{+}\right)$on $S^{2}(\mathbb{D})$ for all $\lambda \in \mathcal{A}_{r}$. Since the class $\mathcal{U} C_{+}$is preserved by compact perturbations we deduce that $\frac{1+r^{2}}{1-r^{2}} C_{\varphi_{r}}-\frac{r}{1-r^{2}}\left(M_{z}^{*}+M_{z}\right) C_{\varphi_{r}}-\lambda I$ is a universal operator on $S^{2}(\mathbb{D})$. 
We include an explicit example of a non-universal composition operator, for which the necessary condition (1) from Theorem 2.2 holds.

Example 3.2. Fix $0<s<1$ and let $\phi: \mathbb{D} \rightarrow \mathbb{D}$ be the hyperbolic non-automorphism defined by $\phi(z)=s z+1-s$. Then

$$
\sigma_{e}^{+}\left(C_{\phi} ; H^{2}(\mathbb{D})\right)=\left\{\lambda \in \mathbb{C}:|\lambda| \leq s^{-1 / 2}\right\},
$$

but $C_{\phi}$ is not universal on $H^{2}(\mathbb{D})$.

In fact, the argument in [16, Thm. 8] implies that for each $\lambda=s^{p+i q}$ with $p>-1 / 2$ and $q \in \mathbb{R}$ (so that $\left.0<|\lambda|<s^{-1 / 2}\right)$ the functions $f_{n}(z)=\exp ((p+i(q+2 n \pi t)) \log (1-z))$, where $t=1 / \log (s)$, are linearly independent in $H^{2}(\mathbb{D}) \cap \operatorname{Ker}\left(C_{\phi}-\lambda I\right)$ for $n \in \mathbb{Z}$. Here log is the principal branch of the natural logarithm. This implies that $(4)$ holds since $\sigma_{e}^{+}\left(C_{\phi} ; H^{2}(\mathbb{D})\right)$ is a closed set. Clearly $C_{\phi}$ is an injective operator and thus not universal. Finally, to obtain an example where 0 belongs to the point spectrum of the operator one extends $C_{\phi}$ to $\left(\begin{array}{cc}C_{\phi} & 0 \\ 0 & 0\end{array}\right)$ on $H^{2}(\mathbb{D}) \oplus[x]$, where $[x]$ denotes a 1-dimensional space.

Recently composition operators have also been studied on the Hardy space and the weighted Bergman spaces of the upper half-plane $\Pi^{+}=\{z \in \mathbb{C}: \operatorname{Im} z>0\}$, where new phenomena occur (see e.g. $[19,10,11]$ ). Recall that the analytic function $F: \Pi^{+} \rightarrow \mathbb{C}$ belongs to the Hardy space $H^{2}\left(\Pi^{+}\right)$if

$$
\|F\|_{H^{2}\left(\Pi^{+}\right)}^{2}=\sup _{y>0} \int_{-\infty}^{\infty}|F(x+i y)|^{2} d x<\infty,
$$

and to the weighted Bergman space $\mathcal{A}_{\alpha}^{2}\left(\Pi^{+}\right)$, for $\alpha>-1$, if

$$
\|F\|_{\mathcal{A}_{\alpha}^{2}\left(\Pi^{+}\right)}^{2}=\int_{0}^{\infty} \int_{-\infty}^{\infty}|F(x+i y)|^{2} y^{\alpha} \frac{d x d y}{\pi}<\infty .
$$

Let $\tau$ be a hyperbolic automorphism of $\Pi^{+}$, that is, $\tau(w)=\mu w+w_{0}$, where $w_{0} \in \mathbb{R}$ and $\mu \in(0,1) \cup(1, \infty)$. It follows from [10, Thm. 3.1] respectively [11, Thm. 3.4] that the composition operator $C_{\tau}$ is bounded on $H^{2}\left(\Pi^{+}\right)$and on $\mathcal{A}_{\alpha}^{2}\left(\Pi^{+}\right)$, for all $\alpha>-1$. It is natural to ask whether there is an analogue of the theorem of Nordgren, Rosenthal and Wintrobe on these spaces.

Proposition 3.3. The operator $C_{\tau}-\lambda I$ is not universal on $H^{2}\left(\Pi^{+}\right)$or $\mathcal{A}_{\alpha}^{2}\left(\Pi^{+}\right)$, where $\alpha>-1$, for any $\lambda \in \mathbb{C}$ and any hyperbolic automorphism $\tau$ of $\Pi^{+}$.

Proof. The claim follows from Corollary 2.4 and the spectral results

$$
\sigma\left(C_{\tau} ; H^{2}\left(\Pi^{+}\right)\right)=\left\{\lambda \in \mathbb{C}:|\lambda|=\mu^{-1 / 2}\right\},
$$

see [20, Thm. 2.12], respectively

$$
\sigma\left(C_{\tau} ; \mathcal{A}_{\alpha}^{2}\left(\Pi^{+}\right)\right)=\left\{\lambda \in \mathbb{C}:|\lambda|=\mu^{-(\alpha+2) / 2}\right\}
$$

for $\alpha>-1$, see [30, Thm. 1.2]. Here $\tau(w)=\mu w+w_{0}$ and $\mu \in(0,1) \cup(1, \infty)$ as above. 


\section{EXAMPLES OF UNIVERSAL COMMUTING PAIRS}

Recently Müller [22] introduced a notion of universality for commuting pairs of operators (and more generally, for commuting $n$-tuples). Let $H$ be a separable infinite-dimensional Hilbert space. The commuting pair $\left(U_{1}, U_{2}\right) \in \mathcal{L}(H)^{2}$ is said to be universal if for each commuting pair $\left(S_{1}, S_{2}\right) \in \mathcal{L}(H)^{2}$ there is a constant $c \neq 0$ and a subspace $M \subset H$, invariant for both $U_{1}$ and $U_{2}$, so that the pairs $\left(U_{1 \mid M}, U_{2 \mid M}\right)$ and $\left(c S_{1}, c S_{2}\right)$ are similar, that is, there is an isomorphism $V: H \rightarrow M$ such that $U_{1} V=c V S_{1}$ and $U_{2} V=c V S_{2}$.

If $\left(U_{1}, U_{2}\right)$ is a universal commuting pair, then $\operatorname{dim}\left(\operatorname{Ker}\left(U_{1}\right) \cap \operatorname{Ker}\left(U_{2}\right)\right)=\infty$, and both $U_{1}$ and $U_{2}$ have to be universal operators for $H$. Müller [22, Thm. 3] obtained a version of Caradus' condition for the universality of commuting pairs $\left(U_{1}, U_{2}\right)$, which we recall next in the special case where $U_{1}, U_{2}$ are surjections, see [22, Cor. 8].

(M) Let $U_{1}, U_{2} \in \mathcal{L}(H)$ be commuting surjections, such that

(i) $\operatorname{dim}\left(\operatorname{Ker}\left(U_{1}\right) \cap \operatorname{Ker}\left(U_{2}\right)\right)=\infty$, and

(ii) $\operatorname{Ker}\left(U_{1} U_{2}\right)=\operatorname{Ker}\left(U_{1}\right)+\operatorname{Ker}\left(U_{2}\right)$.

Then $\left(U_{1}, U_{2}\right) \in \mathcal{L}(H)^{2}$ is a universal commuting pair.

The following concrete example of a universal commuting pair is contained in [22, Examples 9]: Let $H$ be a separable infinite-dimensional Hilbert space and $K=\ell^{2}\left(\mathbb{Z}_{+}^{2}, H\right)$, the space of double-indexed sequences with values in $H$. Define $U_{i} \in \mathcal{L}(K)$ by $U_{i} f(\alpha)=f\left(\alpha+\beta_{i}\right)$ for $\alpha \in \mathbb{Z}_{+}^{2}$ and $f \in \ell^{2}\left(\mathbb{Z}_{+}^{2}, H\right)$ and $i=1,2$, where $\beta_{1}=(1,0)$ and $\beta_{2}=(0,1)$ in $\mathbb{Z}_{+}^{2}$. Then $\left(U_{1}, U_{2}\right) \in \mathcal{L}(K)^{2}$ is a universal commuting pair. (Alternatively, $U_{i}=M_{z_{i}}^{*}$ for $i=1,2$, where $M_{z_{i}}$ denotes multiplication by the variable $z_{i}$ in the vector-valued Hardy space $H^{2}\left(\mathbb{D}^{2}, H\right)$ for $\left(z_{1}, z_{2}\right) \in \mathbb{D}^{2}$.)

In this section we are mainly interested in obtaining further concrete examples of universal commuting pairs, since it turns out that such examples are rather more difficult to write down explicitly compared to the class $\mathcal{U}(H)$. Our first observations and examples illustrate some of the obstructions, apart from the technical fact that condition (M) requires knowledge of $\operatorname{Ker}\left(U_{1}\right)$ and $\operatorname{Ker}\left(U_{2}\right)$. We begin by noting that there is a kind of algebraic independence between $U_{1}$ and $U_{2}$ for universal pairs $\left(U_{1}, U_{2}\right)$. For this let $\{T\}^{\prime}=\{S \in \mathcal{L}(H): S T=T S\}$ stand for the commutant of $T$.

Proposition 4.1. Let $H$ is a separable infinite-dimensional Hilbert space and $T \in \mathcal{L}(H)$.

(i) if $S \in\{T\}^{\prime}$, then $(T, S T)$ is not a universal commuting pair. In particular, $(T, p(T) T)$ is not a universal commuting pair for any complex polynomial $p(z)=a_{1} z+\ldots+a_{n} z^{n}$ satisfying $p(0)=0$ where $p(T)=a_{1} T+\ldots+a_{n} T^{n}$.

(ii) $\left(T^{m}, T^{n}\right)$ is not a universal commuting pair for any $m, n \in \mathbb{N}$.

Proof. (i) Consider $\left(0, I_{H}\right) \in \mathcal{L}(H)^{2}$. If $(T, S T)$ is a universal pair, then corresponding to the pair $\left(0, I_{H}\right) \in \mathcal{L}(H)^{2}$ there is an infinite-dimensional subspace $M \subset H$ invariant under $T$, and $c \neq 0$, so that $\left(T_{\mid M}, S T_{\mid M}\right)$ and $\left(0, c I_{H}\right)$ are similar. However, the similarity of $T_{\mid M}$ and 0 implies that $M \subset \operatorname{Ker}(T)$, so that $S T_{\mid M}$ cannot be similar to $c I_{H}$.

For part (ii) observe that one may assume that $m<n$ by symmetry, whence one may argue as in part (i).

The following example looks at simple ways to construct universal pairs starting from given universal operators $U, V \in \mathcal{U}(H)$. 
Example 4.1. (i) Suppose that $U, V \in \mathcal{L}(H)$ satisfy condition (C), and

$$
U_{0}=\left(\begin{array}{cc}
U & 0 \\
0 & I_{H}
\end{array}\right), \quad V_{0}=\left(\begin{array}{cc}
I_{H} & 0 \\
0 & V
\end{array}\right) \in \mathcal{L}(K),
$$

where $K=H \oplus H$. Then $U_{0}$ and $V_{0}$ are commuting surjections on $K$, and $\operatorname{Ker}\left(U_{0}\right)=$ $\operatorname{Ker}(U) \times\{0\}$ and $\operatorname{Ker}\left(V_{0}\right)=\{0\} \times \operatorname{Ker}(V)$. Hence the pair $\left(U_{0}, V_{0}\right) \in(\mathcal{L}(K))^{2}$ is not universal, since $\operatorname{Ker}\left(U_{0}\right) \cap \operatorname{Ker}\left(V_{0}\right)=\{(0,0)\}$. (Note however that $\operatorname{Ker}\left(U_{0} V_{0}\right)=\operatorname{Ker}\left(U_{0}\right)+\operatorname{Ker}\left(V_{0}\right)$ in this case.)

(ii) Suppose that $\left(U_{1}, U_{2}\right)$ and $\left(V_{1}, V_{2}\right)$ are commuting pairs of surjections that satisfy condition $(\mathrm{M})$, and put

$$
U=\left(\begin{array}{cc}
U_{1} & 0 \\
0 & V_{1}
\end{array}\right), \quad V=\left(\begin{array}{cc}
U_{2} & 0 \\
0 & V_{2}
\end{array}\right) \in \mathcal{L}(K) .
$$

In this case $(U, V) \in \mathcal{L}(K)^{2}$ is a universal commuting pair. In fact, $(U, V)$ also satisfies $(\mathrm{M})$, since it is not difficult to check that

$$
\operatorname{Ker}(U V)=\left(\operatorname{Ker}\left(U_{1}\right)+\operatorname{Ker}\left(U_{2}\right)\right) \times\left(\operatorname{Ker}\left(V_{1}\right)+\operatorname{Ker}\left(V_{2}\right)\right)=\operatorname{Ker}(U)+\operatorname{Ker}(V) .
$$

We next look for a non-commutative version of the example from [22]. Let $H$ be a separable infinite-dimensional Hilbert space and $\mathcal{C}_{2}(H)$ the space of Hilbert-Schmidt operators on $H$ equipped with the Hilbert-Schmidt norm $\|\cdot\|_{2}$. Recall that $T \in \mathcal{C}_{2}(H)$ if there is an orthonormal basis $\left(f_{n}\right)$ of $H$ such that $\sum_{n=1}^{\infty}\left\|T f_{n}\right\|^{2}<\infty$, where

$$
\|T\|_{2}=\left(\sum_{n=1}^{\infty}\left\|T f_{n}\right\|^{2}\right)^{1 / 2}
$$

is independent of the basis. Then $\left(\mathcal{C}_{2}(H),\|\cdot\|_{2}\right)$ is a separable Hilbert space, and $\|U S V\|_{2} \leq$ $\|U\| \cdot\|V\| \cdot\|S\|_{2}$ for $S \in \mathcal{C}_{2}(H)$ and $U, V \in \mathcal{L}(H)$. We refer e.g. to [23, chapter 2.4] for more background on the ideal $\mathcal{C}_{2}(H)$ of $\mathcal{L}(H)$.

Hence the multiplication maps $L_{U}$ and $R_{U}$ are bounded operators $\mathcal{C}_{2}(H) \rightarrow \mathcal{C}_{2}(H)$, where $L_{U}(S)=U S$ and $R_{U}(S)=S U$ for any $U \in \mathcal{L}(H)$ and $S \in \mathcal{C}_{2}(H)$. Clearly $\left(L_{U}, R_{V}\right) \in$ $\mathcal{L}\left(\mathcal{C}_{2}(H)\right)^{2}$ is a commuting pair for any $U, V \in \mathcal{L}(H)$, and we are interested in the universality of $\left(L_{U}, R_{V}\right)$ on $\mathcal{C}_{2}(H)$. Let $B$ be the standard backward shift on $\ell^{2}=\ell^{2}\left(\mathbb{Z}_{+}\right)$, that is, $B\left(x_{0}, x_{1}, \ldots\right)=\left(x_{1}, x_{2}, \ldots\right)$ for $\left(x_{j}\right) \in \ell^{2}$. It turns out that $\left(L_{B}, R_{B^{*}}\right)$ is not a universal pair (see part (ii) of Theorem 4.2), and to obtain universal pairs $\left(L_{U}, R_{V}\right)$ we will consider the vector-valued direct $\ell^{2}$-sum $\mathcal{H}=\ell^{2}\left(\mathbb{Z}_{+}, H\right)=\left(\oplus_{n \in \mathbb{Z}_{+}} H\right)_{\ell^{2}}$, where $H$ is a fixed separable infinite-dimensional Hilbert space. Let $B_{\infty}$ be the backward shift of infinite multiplicity on $\mathcal{H}$, so that $B_{\infty}^{*}$ is the corresponding forward shift on $\mathcal{H}$.

The following result contains the main example of this section. We will use $u \otimes v$ for given $u, v \in H$ to denote the rank-1 operator $x \mapsto\langle x, v\rangle u$ on $H$.

Theorem 4.2. (i) $L_{B}, R_{B^{*}} \in \mathcal{U}\left(\mathcal{C}_{2}\left(\ell^{2}\right)\right)$ and $L_{B_{\infty}}, R_{B_{\infty}^{*}} \in \mathcal{U}\left(\mathcal{C}_{2}(\mathcal{H})\right)$.

(ii) $\left(L_{B}, R_{B^{*}}\right)$ is not a universal pair on $\mathcal{C}_{2}\left(\ell^{2}\right)$.

(iii) $\left(L_{B_{\infty}}, R_{B_{\infty}^{*}}\right)$ is a universal pair on $\mathcal{C}_{2}(\mathcal{H})$.

Proof. (i) We check that $L_{B}$ and $R_{B^{*}}$ satisfy condition $(\mathrm{C})$ on $\mathcal{C}_{2}\left(\ell^{2}\right)$. In fact, if $\left(e_{n}\right)$ is the standard unit vector basis of $\ell^{2}$ then $L_{B}\left(e_{0} \otimes e_{n}\right)=B e_{0} \otimes e_{n}=0$ for any $n \in \mathbb{Z}_{+}$, so that 
$\operatorname{Ker}\left(L_{B}\right)$ is infinite-dimensional. Moreover, $\operatorname{Ran}\left(L_{B}\right)=\mathcal{C}_{2}\left(\ell^{2}\right)$ since $B B^{*}=I_{H}$, and the argument for $R_{B^{*}}$ is similar.

The universality of $L_{B_{\infty}}$ and $R_{B_{\infty}^{*}}$ on $\mathcal{C}_{2}(\mathcal{H})$ follows from part (iii) (alternatively, one may also modify the preceding argument as in the proof of (iii)).

(ii) Recall that $\left(e_{m} \otimes e_{n}\right)_{m, n \in \mathbb{Z}_{+}}$is an orthonormal basis of $\mathcal{C}_{2}\left(\ell^{2}\right)$. It follows from the identities $L_{B}\left(e_{m} \otimes e_{n}\right)=B e_{m} \otimes e_{n}$ and $R_{B^{*}}\left(e_{m} \otimes e_{n}\right)=e_{m} \otimes B e_{n}$ that $\operatorname{Ker}\left(L_{B}\right)=\left[e_{0} \otimes e_{n}\right.$ : $\left.n \in \mathbb{Z}_{+}\right]$and $\operatorname{Ker}\left(R_{B^{*}}\right)=\left[e_{n} \otimes e_{0}: n \in \mathbb{Z}_{+}\right]$. Here $[A]$ denotes the closed linear span of the set $A \subset \mathcal{C}_{2}\left(\ell^{2}\right)$. In particular, $\operatorname{Ker}\left(L_{B}\right) \cap \operatorname{Ker}\left(R_{B^{*}}\right)=\left[e_{0} \otimes e_{0}\right]$ is 1-dimensional, so that $\left(L_{B}, R_{B^{*}}\right)$ can not be a universal pair.

(iii) We will verify that condition (M) holds. Towards this note first that $L_{B_{\infty}}$ and $R_{B_{\infty}^{*}}$ are surjections on $\mathcal{C}_{2}(\mathcal{H})$, since $B_{\infty} B_{\infty}^{*}=I_{\mathcal{H}}$ implies that $L_{B_{\infty}}\left(B_{\infty}^{*} S\right)=S$ and $R_{B_{\infty}^{*}}\left(S B_{\infty}\right)=S$ for any $S \in \mathcal{C}_{2}(\mathcal{H})$.

To compute the kernels of $L_{B_{\infty}}$ and $R_{B_{\infty}^{*}}$ we need the fact that any $S \in \mathcal{C}_{2}(\mathcal{H})$ is uniquely determined by its operator-matrix components $S_{i, j}=P_{i} S J_{j} \in \mathcal{C}_{2}(H)$ for $i, j \in \mathbb{Z}_{+}$. Here $P_{i}$ is the orthogonal projection $\mathcal{H} \rightarrow H$ onto the $i$ :th copy of $H$, and $J_{j}: H \rightarrow \mathcal{H}$ the canonical inclusion from the $j$-th copy. One deduces from the definition of $S_{i, j}$ and the identity $L_{B_{\infty}}(v \otimes u)=B_{\infty} v \otimes u$ for $u, v \in \mathcal{H}$ that

$$
L_{B_{\infty}}(S)=\left(\begin{array}{ccc}
S_{1,0} & S_{1,1} & \cdots \\
S_{2,0} & S_{2,1} & \cdots \\
\vdots & \vdots & \ddots
\end{array}\right)
$$

for $S=\left(S_{i, j}\right)$, so that by uniqueness

$$
\operatorname{Ker}\left(L_{B_{\infty}}\right)=\left\{S=\left(S_{i, j}\right) \in \mathcal{C}_{2}(\mathcal{H}): S_{i, j}=0 \text { for } i>0\right\} .
$$

Similarly, the identity $R_{B_{\infty}^{*}}(v \otimes u)=v \otimes B_{\infty} u$ for $u, v \in \mathcal{H}$ yields that

$$
R_{B_{\infty}^{*}}(S)=\left(\begin{array}{ccc}
S_{0,1} & S_{0,2} & \cdots \\
S_{1,1} & S_{1,2} & \cdots \\
\vdots & \vdots & \ddots
\end{array}\right),
$$

whence

$$
\operatorname{Ker}\left(R_{B_{\infty}^{*}}\right)=\left\{S=\left(S_{i, j}\right) \in \mathcal{C}_{2}(\mathcal{H}): S_{i, j}=0 \text { for } j>0\right\} .
$$

In particular, we get that

$$
\operatorname{Ker}\left(L_{B_{\infty}}\right) \cap \operatorname{Ker}\left(R_{B_{\infty}^{*}}\right)=\left\{S=\left(S_{i, j}\right) \in \mathcal{C}_{2}(\mathcal{H}): S_{i, j}=0 \text { for } i>0 \text { or } j>0\right\}
$$

is infinite-dimensional, since the operator $S_{0,0} \in \mathcal{C}_{2}(H)$ can be chosen freely. Finally, we need to verify that

$$
\operatorname{Ker}\left(L_{B_{\infty}} R_{B_{\infty}^{*}}\right)=\operatorname{Ker}\left(L_{B_{\infty}}\right)+\operatorname{Ker}\left(R_{B_{\infty}^{*}}\right) .
$$

However, (7) follows from (5) and (6), the identity $L_{B_{\infty}} R_{B_{\infty}^{*}}(v \otimes u)=B_{\infty} v \otimes B_{\infty} u$ for $u, v \in \mathcal{H}$, the observation that

$$
L_{B_{\infty}} R_{B_{\infty}^{*}}(S)=\left(\begin{array}{ccc}
S_{1,1} & S_{1,2} & \cdots \\
S_{2,1} & S_{2,2} & \cdots \\
\vdots & \vdots & \ddots
\end{array}\right)
$$


as well as the fact that

$$
\left(\begin{array}{ccc}
S_{0,0} & S_{0,1} & \cdots \\
S_{1,0} & 0 & \cdots \\
\vdots & \vdots & \ddots
\end{array}\right)=\left(\begin{array}{ccc}
S_{0,0} & S_{0,1} & \cdots \\
0 & 0 & \cdots \\
\vdots & \vdots & \ddots
\end{array}\right)+\left(\begin{array}{ccc}
0 & 0 & \cdots \\
S_{1,0} & 0 & \cdots \\
\vdots & \vdots & \ddots
\end{array}\right)
$$

is the sum of two well-defined Hilbert-Schmidt operators for any $S=\left(S_{i, j}\right) \in \mathcal{C}_{2}(\mathcal{H})$, see e.g. the proof of $[17,1 . c .8]$.

We conclude from condition $(\mathrm{M})$ that $\left(L_{B_{\infty}}, R_{B_{\infty}^{*}}\right)$ is a universal pair.

Remarks. By a straightforward modification of the argument in part (iii) one may also show that $\left(L_{\left(B_{\infty}\right)^{m}}, R_{\left(B_{\infty}^{*}\right)^{n}}\right)$ is a universal pair on $\mathcal{C}_{2}(\mathcal{H})$ for any $m, n \in \mathbb{N}$. We do not know explicit conditions on $(U, V) \in(\mathcal{L}(H))^{2}$ which ensure that $\left(L_{U}, R_{V}\right)$ is a universal pair on $\mathcal{C}_{2}(H)$.

For our last examples we return to the setting (and notations) from Section 3 related to composition operators on $H^{2}(\mathbb{D})$ associated to hyperbolic automorphisms of $\mathbb{D}$. Recall that $C_{\varphi_{r}}-\lambda I$ and $C_{\varphi_{s}}-\mu I$ commute for any $0<r, s<1$ and $\lambda, \mu \in \mathbb{C}$. This follows from the fact that

$$
\varphi_{r} \circ \varphi_{s}=\varphi_{t}=\varphi_{s} \circ \varphi_{r}
$$

where $t=\frac{r+s}{1+r s}$. The result of Nordgren, Rosenthal and Wintrobe [24] suggests the following natural question.

Problem. Are there universal pairs of the form

$$
\left(C_{\varphi_{r}}-\lambda I, C_{\varphi_{s}}-\mu I\right) \in \mathcal{L}\left(H^{2}(\mathbb{D})\right)^{2},
$$

for some $0<r, s<1, \lambda \in \mathcal{A}_{r}$ and $\mu \in \mathcal{A}_{s}$ ?

We first note some obvious restrictions in view of Proposition 4.1.

Example 4.3. (i) The pair $\left(C_{\varphi_{r}}-\lambda I, C_{\varphi_{r}}-\mu I\right)$ is not universal for any $0<r<1$ and $\lambda \neq \mu$. In fact, in this case $\operatorname{Ker}\left(C_{\varphi_{r}}-\lambda I\right) \cap \operatorname{Ker}\left(C_{\varphi_{r}}-\mu I\right)=\{0\}$ once $\lambda \neq \mu$.

(ii) Let $0<r<1$. By $(8)$ there is $r_{n} \in(0,1)$ such that $\varphi_{r}^{n}=\varphi_{r_{n}}$, where $\varphi_{r}^{n}=\varphi_{r} \circ \ldots \circ \varphi_{r}$ ( $n$-fold composition). Then $\left(C_{\varphi_{r}}-\lambda I, C_{\varphi_{r_{n}}}-\lambda^{n} I\right)$ is not a universal pair for any $n \geq 2$ and $\lambda \in \mathcal{A}_{r}$. Indeed, to see this we write $C_{\varphi_{r_{n}}}-\lambda^{n} I=S\left(C_{\varphi_{r}}-\lambda I\right)$, where $S$ commutes with $C_{\varphi_{r}}$, and apply Proposition 4.1.

In our final example we use the recent approach of Cowen and Gallardo-Gutiérrez [8] to the universality result by Nordgren, Rosenthal and Wintrobe in order to analyse more carefully a pair, which shows subtler obstructions related to the existence of NRW-pairs.

Example 4.4. There are $r, s \in(0,1)$ and respective eigenvalues $\lambda \in \mathcal{A}_{r}, \mu \in \mathcal{A}_{s}$ such that $\operatorname{Ker}\left(C_{\varphi_{r}}-\lambda I\right) \cap \operatorname{Ker}\left(C_{\varphi_{s}}-\mu I\right)$ is infinite-dimensional, but condition (M) fails to hold for the pair $\left(C_{\varphi_{r}}-\lambda I, C_{\varphi_{s}}-\mu I\right)$.

Proof. Fix $0<r<1$ and consider the positive eigenvalue $\lambda=\left(\frac{1-r}{1+r}\right)^{-u} \in \mathcal{A}_{r}$, where $0<u<$ $1 / 2$. It follows from the proofs of Lemma 7.3 and Theorem 7.4 in [9] that the functions

$$
f_{n}(z)=\exp \left((u+i n 2 \pi a) \log \frac{1+z}{1-z}\right)
$$


where $a=\left(\log \frac{1-r}{1+r}\right)^{-1}$ and $n \in \mathbb{Z}$, forms a linearly independent family of eigenfunctions for $C_{\varphi_{r}}$ in $H^{2}(\mathbb{D})$ associated with the eigenvalue $\lambda$. Here the logarithm refers to the principal branch. Let $r<s<1$ and consider $\mu=\left(\frac{1-s}{1+s}\right)^{-u} \in \mathcal{A}_{s}$. As above

$$
g_{m}(z)=\exp \left((u+i m 2 \pi b) \log \frac{1+z}{1-z}\right) \text {, }
$$

where $b=\left(\log \frac{1-s}{1+s}\right)^{-1}$ and $m \in \mathbb{Z}$, are linearly independent eigenfunctions of $C_{\varphi_{s}}$ for the eigenvalue $\mu$. Moreover, we select $r$ and $s$ so that

$$
\frac{n}{m}=\frac{b}{a}=\frac{\log \frac{1+r}{1-r}}{\log \frac{1+s}{1-s}}
$$

holds for infinitely many pairs $n, m \in \mathbb{Z} \backslash\{0\}$. This ensures that $\operatorname{Ker}\left(C_{\varphi_{r}}-\lambda I\right) \cap \operatorname{Ker}\left(C_{\varphi_{s}}-\mu I\right)$ is infinite-dimensional by comparing the above eigenfunctions.

Recall from [4, Thm. 5] (see also [8]) that there is an analytic covering map $\psi_{r}: \mathbb{D} \rightarrow \mathcal{A}_{r}$, such that $C_{\varphi_{r}}-\lambda I$ and $T_{\psi_{r}-\lambda}^{*}$ are similar operators on $H^{2}(\mathbb{D})$, where $T_{\psi_{r}-\lambda} \in \mathcal{L}\left(H^{2}(\mathbb{D})\right)$ is the analytic Toeplitz operator $f \mapsto\left(\psi_{r}-\lambda\right) f$. Indeed,

$$
\psi_{r}(z)=\left(\frac{1-z}{1+z}\right)^{i t_{r} / \pi}
$$

where $t_{r}=-\log \left(\frac{1-r}{1+r}\right)>0$. Moreover, $C_{\varphi_{s}}-\mu I$ and $T_{\psi_{s}-\mu}^{*}$ are similar operators on $H^{2}(\mathbb{D})$ for the covering map $\psi_{s}: \mathbb{D} \rightarrow \mathcal{A}_{s}$, where $\psi_{s}(z)=\left(\frac{1-z}{1+z}\right)^{i t_{s} / \pi}$ and $t_{s}=-\log \left(\frac{1-s}{1+s}\right)$.

By standard duality, the dual version of part (ii) of condition $(\mathrm{M})$ for the pair $\left(T_{\psi_{r}-\lambda}^{*}, T_{\psi_{s}-\mu}^{*}\right)$ is the requirement that

$$
\operatorname{Ran}\left(T_{\psi_{s}-\mu} T_{\psi_{r}-\lambda}\right)=\operatorname{Ran}\left(T_{\psi_{r}-\lambda}\right) \cap \operatorname{Ran}\left(T_{\psi_{s}-\mu}\right) .
$$

Note for this that all the ranges are closed, since the adjoints are onto maps by similarity.

We claim that condition (10) does not hold. Towards this consider the standard factorisation $\psi_{r}-\lambda=B_{1} S_{1} F_{1}$ into a Blaschke product $B_{1}$ containing the zeroes of the function (counting multiplicity), a singular inner function $S_{1}$ and an outer function $F_{2}$. Let $\psi_{s}-\mu=B_{2} S_{2} F_{2}$ be the analogous factorisation for $\psi_{s}-\mu$. It is not difficult to check from (9) and the explicit form of $\psi_{r}$ and $\psi_{s}$ that the functions $\psi_{r}-\lambda$ and $\psi_{s}-\mu$ have infinitely many simple common zeroes. Let $B_{1}=B_{0} B_{3}$ and $B_{2}=B_{0} B_{4}$, where $B_{0}$ is the Blaschke product which contains the common zeroes.

To conclude the argument consider the function $g=B_{0} B_{3} B_{4} S_{1} F_{1} S_{2} F_{2}$. Observe that $g \in H^{2}(\mathbb{D})$ since the maps $\psi_{r}-\lambda$ and $\psi_{s}-\mu$, and hence also $S_{1} F_{1}$ and $S_{2} F_{2}$, belong to $H^{\infty}$ (see [29, Thm. 17.9], for instance). Clearly $g \in \operatorname{Ran}\left(T_{\psi_{r}-\lambda}\right) \cap \operatorname{Ran}\left(T_{\psi_{s}-\mu}\right)$ by inspection. However, the Blaschke product containing the zeroes of $\left(\psi_{r}-\lambda\right)\left(\psi_{s}-\mu\right)$ already has the form $B_{0}^{2} B_{3} B_{4}$, so that $g \notin \operatorname{Ran}\left(T_{\psi_{s}-\mu} T_{\psi_{r}-\lambda}\right)$ in view of the uniqueness of the factorisation.

Remarks. One may verify from (9) that the pair $\left(C_{\varphi_{r}}-\lambda I, C_{\varphi_{s}}-\mu I\right)$ studied in Example 4.4 has the property that there is $p, q \in \mathbb{N}$ with $p<q$, for which $C_{\varphi_{r}}^{q}-\lambda^{q} I=C_{\varphi_{s}}^{p}-\mu^{p} I$. However, we do not have general results which would exclude such a property for universal pairs. 


\section{ACKNowledGements}

The authors thank Isabelle Chalendar for drawing the attention to reference [22], and Pekka Nieminen for useful comments. The first author is grateful to Eva Gallardo-Gutiérrez for several illuminating discussions on universal operators during the past years.

This paper is part of the first author's PhD thesis, which is supervised by the second author and Pekka Nieminen.

\section{REFERENCES}

[1] S. R. Caradus. Universal operators and invariant subspaces. Proc. Amer. Math. Soc., 23:526-527, 1969.

[2] S. R. Caradus, W. E. Pfaffenberger, and Bertram Yood. Calkin algebras and algebras of operators on Banach spaces. Marcel Dekker, Inc., New York, 1974. Lecture Notes in Pure and Applied Mathematics, Vol. 9.

[3] Isabelle Chalendar and Jonathan R. Partington. Modern approaches to the invariant-subspace problem, volume 188 of Cambridge Tracts in Mathematics. Cambridge University Press, Cambridge, 2011.

[4] Carl C. Cowen and Eva A. Gallardo-Gutiérrez. Unitary equivalence of one-parameter groups of Toeplitz and composition operators. J. Funct. Anal., 261(9):2641-2655, 2011.

[5] Carl C. Cowen and Eva A. Gallardo-Gutiérrez. Consequences of universality among Toeplitz operators. J. Math. Anal. Appl., 432(1):484-503, 2015.

[6] Carl C. Cowen and Eva A. Gallardo-Gutiérrez. An introduction to Rota's universal operators: properties, old and new examples and future issues. Concr. Oper., 3:43-51, 2016.

[7] Carl C. Cowen and Eva A. Gallardo-Gutiérrez. Rota's universal operators and invariant subspaces in Hilbert spaces. J. Funct. Anal., 271(5):1130-1149, 2016.

[8] Carl C. Cowen and Eva A. Gallardo-Gutiérrez. A new proof of a Nordgren, Rosenthal and Wintrobe theorem on universal operators. Problems and Recent Methods in Operator Theory (Memphis, TN, 2015), 97-102, Contemp. Math.. 687, Amer. Math. Soc., 2017.

[9] Carl C. Cowen and Barbara D. MacCluer. Composition operators on spaces of analytic functions. Studies in Advanced Mathematics. CRC Press, Boca Raton, FL, 1995.

[10] Sam Elliott and Michael T. Jury. Composition operators on Hardy spaces of a half-plane. Bull. Lond. Math. Soc., 44(3):489-495, 2012.

[11] Sam J. Elliott and Andrew Wynn. Composition operators on weighted Bergman spaces of a half-plane. Proc. Edinb. Math. Soc. (2), 54(2):373-379, 2011.

[12] Eva A. Gallardo-Gutiérrez and Pamela Gorkin. Minimal invariant subspaces for composition operators. J. Math. Pures Appl. (9), 95(3):245-259, 2011.

[13] Eva A. Gallardo-Gutiérrez and Riikka Schroderus. The spectra of linear fractional composition operators on weighted Dirichlet spaces. J. Funct. Anal., 271(3):720-745, 2016.

[14] Katherine Heller. Adjoints of linear fractional composition operators on $S^{2}(\mathbb{D})$. J. Math. Anal. Appl., 394(2):724-737, 2012.

[15] William M. Higdon. The spectra of composition operators from linear fractional maps acting upon the Dirichlet space. J. Funct. Anal., 220(1):55-75, 2005.

[16] Paul R. Hurst. Relating composition operators on different weighted Hardy spaces. Arch. Math. (Basel), 68(6):503-513, 1997.

[17] Joram Lindenstrauss and Lior Tzafriri. Classical Banach spaces. I. Springer-Verlag, Berlin-New York, 1977. Sequence spaces, Ergebnisse der Mathematik und ihrer Grenzgebiete, Vol. 92.

[18] Valentin Matache. On the minimal invariant subspaces of the hyperbolic composition operator. Proc. Amer. Math. Soc., 119(3):837-841, 1993.

[19] Valentin Matache. Composition operators on Hardy spaces of a half-plane. Proc. Amer. Math. Soc., 127(5):1483-1491, 1999.

[20] Valentin Matache. Invertible and normal composition operators on the Hilbert Hardy space of a half-plane. Concr. Oper., 3:77-84, 2016.

[21] Vladimir Müller. Spectral theory of linear operators and spectral systems in Banach algebras, volume 139 of Operator Theory: Advances and Applications. Birkhäuser Verlag, Basel, 2003.

[22] Vladimir Müller. Universal $n$-tuples of operators. Math. Proc. R. Ir. Acad., 113A(2):143-150, 2013.

[23] Gerard J. Murphy. $C^{*}$-algebras and operator theory. Academic Press, Inc., Boston, MA, 1990. 
[24] Eric Nordgren, Peter Rosenthal, and F. S. Wintrobe. Invertible composition operators on $H^{p}$. J. Funct. Anal., 73(2):324-344, 1987.

[25] Jonathan R. Partington and Elodie Pozzi. Universal shifts and composition operators. Oper. Matrices, 5(3):455-467, 2011.

[26] Elodie Pozzi. Universality of weighted composition operators on $L^{2}([0,1])$ and Sobolev spaces. Acta Sci. Math. (Szeged), 78(3-4):609-642, 2012.

[27] Marvin Rosenblum and James Rovnyak. Hardy classes and operator theory. Dover Publications, Inc., Mineola, NY, 1997. Corrected reprint of the 1985 original.

[28] G. C. Rota. Note on the invariant subspaces of linear operators. Rend. Circ. Mat. Palermo (2), 8:182-184, 1959.

[29] Walter Rudin. Real and complex analysis. McGraw-Hill Book Co., New York, third edition, 1987.

[30] Riikka Schroderus. Spectra of linear fractional composition operators on the Hardy and weighted Bergman spaces of the half-plane. J. Math. Anal. Appl., 447(2):817-833, 2017.

[31] Joel H. Shapiro. Composition operators and classical function theory. Universitext: Tracts in Mathematics. Springer-Verlag, New York, 1993.

[32] Nina Zorboska. Composition operators on $S_{a}$ spaces. Indiana Univ. Math. J., 39(3):847-857, 1990.

Department of Mathematics and Statistics, University of Helsinki, Box 68, Fi-00014 Helsinki, FINLAND

E-mail address: riikka.schroderus@helsinki.fi

Department of Mathematics and Statistics, University of Helsinki, Box 68, FI-00014 Helsinki, FinLAND

E-mail address: hans-olav.tylli@helsinki.fi 